\section{The AIDS epidemic}

Despite the significant advances in the treatment of AIDS with protease inhibitors and other new drugs, we were reminded at the end of last year that the AIDS epidemic is far from over. A report prepared to coincide with World AIDS Day on 1 December 1997 (Report on the Global HIV/AIDS Epidemic, United Nations, 1997) in fact suggests that there have been serious underestimations made of the epidemic: currently, according to United Nations figures, the prevalence of HIV positivity is $1 \%$ among sexually active individuals on a worldwide scale and 16000 new infections occur each day. These are startling figures and should give cause for concern since much of the new disease occurs in countries with poor economic access to the new therapies. In addition, the UN office indicates that although some of the previously lower figures were based on less accurate data collection, the new figures suggest that a significant proportion of individuals may be unaware that they are HIV positive. Thus, public health campaigns to control the disease may need to rethink their strategies.

Global estimates of HIV/AIDS infection at current infection rates indicate that around 50 million individuals will be infected by the year 2000. The annual rate of AIDS deaths is rising by $50 \%$ per annum and a significant proportion of these individuals are children. In fact, the infection rate among children is as high as $10 \%$ of the sexually active adult rate. AIDS is thus a disease of young people, with serious demographic implications. The UN has initiated a campaign (UNAIDS) to provide access to the new drugs to developing countries particularly certain parts of Africa which appear to be most seriously affected. Indeed, approximately one third of the total caseload worldwide is concentrated in this region.

\section{Creutzfeldt-Jakob disease (CJD) and ocular tissue transplantation}

It has been a long held secret among ophthalmologists that CJD could be transmitted via transplantation of corneal tissue (for review see Hogan and Cavanah, Cornea 1995;14:545-6). However, as is the wont of current press journalistic techniques, which seem to be aimed at inducing public terror, the most recent case of CJD in a patient who had a corneal transplant was firmly placed within the context of the ongoing BSE scare. On 1 December 1997, a press statement confirmed that a 54 year old woman from Scotland, who had died in February 1997 and had donated her organs for transplantation, was confirmed as having CJD. This information had not emerged until nine months after her death and at least six months after each cornea and both scleras had been transplanted into three recipients. The tissue had been processed by the Corneal Transplant Service Eye Bank in Manchester.

Although the diagnosis was of classic (sporadic) CJD there was a certain amount of confusion in the press; the diagnosis was at times erroneously reported as being new variant CJD-that is, the one believed to be the human equivalent of BSE.

This serious adverse event is the subject of a Scottish Office inquiry chaired by $\mathrm{Sir}$ William Stewart which is expected to report early in 1998. In the meantime the response has included the identification and counselling of the three recipients of the donor tissue including consideration of secondary inter vention; the possibility of secondary contamination of instruments used in retrieval, eye banking, and transplantation; measures to minimise the risk of such an event occurring again; measures to limit the risk of secondary contamination by changes in instrument sterilisation policies; and finally in the derivation of guidelines (which do not currently exist) on how to act under such circumstances in future. Clearly, a major aim is the development of a reliable and suitable test which can be used to screen for CJD but whether this is likely to occur in the near future remains to be seen.

\section{Microphthalmia, stem cell factor, and MAP kinase}

Nowadays it is becoming relatively commonplace for the most unexpected associations to occur. Waardenburg's syndrome in which defects in skin, eye (microphthalmos), and ear occur as well as increased susceptibility to infections, osteopetrosis, and mental retardation is caused by a defect in the gene, microphthalmos (Mi). In particular, Mi appears to be involved in mast cell and melanocyte differentiation. Clearly it has been difficult to envisage how such a diverse set of phenotypic characteristics can be mediated through a single gene. However, a recen paper (Hemesath et al, Nature 1998;391:298301) has now uncovered the link. $\mathrm{Mi}$ is the gene for a transcription factor of the leucine zipper type which binds E-box type enhance elements and may function in combination with other family members of the same type. The gene c-kit and its ligand, stem cell facto (steel factor, c-kit ligand) also control certain aspects of melanocyte and mast cell growth and mice with mutations in the gene have pigmentation and stem cell abnormalities. It now appears that activation of $\mathrm{Mi}$ is induced during development under the regulatory control of c-kit. Binding of c-kit to cell surface stem cell factor on melanocytes and mast cells induces expression of $\mathrm{Mi}$ and is mediated via phosphorylation of that ubiquitous kinase MAP kinase, essential to many aspects of cell growth and differentiation. Thus, a common pathway for several aspects of cell and tissue specific differentiation is described. Of interest to developmental ophthalmic scientists are the implications of normal mast cell and melanocyte differentiation to ultimate norma eye growth. The notion that melanocytes and mast cells may release growth factors necessary for full adult ocular growth is intriguing and worthy of investigation perhaps in transgenic models.

\section{Designer anti-inflammatory drugs on the horizon?}

Much tissue destruction is mediated via the proinflammatory cytokines tumour necrosis factor $\alpha(\mathrm{TNF}-\alpha)$ and interleukin 1. This applies particularly to ocular inflammatory disease, both infectious and non-infectious. Several approaches to treating ocular inflammation have been suggested with cytokines as the specific targets. However, for a variety of reasons these have not yet reached the clinic although some very interesting experimental work has been reported using, for instance, $\mathrm{TNF}-\alpha$ receptor fusion proteins. Perhaps a more direct approach is to get to the heart of the machinery - that is, intracellularly, where the cytokines induce inflammatory cell activation via intracellular signals. Both $\mathrm{TNF}-\alpha$ and IL-1 mediate their proinflammatory effects via nuclear factor kappa-B (NF-кB) which is normally inhibited via a set of cytosolic protein inhibitors (ikB). When TNF- $\alpha$ and/or IL-1 bind to the cell they can induce at least 50 genes via $\mathrm{NF}-\kappa \mathrm{B}$. However, precisely how they did this was not clear. In a recent review (Current Biology 1998;8:R19-22), the missing parts of this intracellular signalling pathway have been described following the discovery of several protein kinases which go under the unlikely names of TRADD, TRAF2, and RIP for the TNFr and IRAK and TRAF6 for the IL-1 $r$. These interact with a set of downstream kinases (NIK, IKK-a and IKK-b) which have also been recently discovered and allow the

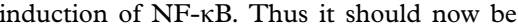
possible to design specific drugs which can be targeted to selectively switch off these second messengers and thus reduce inflammation. For many inflammatory diseases these are exciting new data but especially for ophthalmologists since the prospect of such potent but selective topical anti-inflammatories is very encouraging.

\section{Neurons caught talking to themselves}

Autapses are the name for synapses that neurons form with themselves. This intuitively disturbing finding, at least for neurophysiologists, but suggested at least 100 years ago, who are accustomed to considering unidirectional flow of information, has been confirmed by several studies recently (for review see Current Biology 1998;8:R52). Despite their wide distribution in the CNS the number per neuron is rather low and their function is still unclear. Two suggestions have been made-either they may act as "gates" regulating the flow of information from distal synapses on the neuronal axon while information from proximal synapses is allowed free passage; or it may simply reflect a mechanism of autoinhibition whereby the autaptic synapse at least partially blocks the dendritic input by blocking the $\mathrm{Ca}+$ spike and does so in a very precise phase locked manner which allows fine control of interneuron activity. It is likely the autapses are also transient and may be involved to a degree in neuronal plasticity. 